\title{
Alfabetización Informacional: una vía de acceso a la información confiable ${ }^{1}$
}

\author{
Fredy Mauricio Gutiérrez Valderrama ${ }^{2}$ \\ Institución Educativa Arnulfo Briceño Contreras, Villavicencio, Colombia \\ http://orcid.org/0000-0002-2171-8818 \\ Myriam Cecilia Leguizamón González ${ }^{3}$ \\ Universidad Pedagógica y Tecnológica de Colombia, Colombia \\ http://orcid.org/0000-0003-4549-7156
}

Artículo de investigación https://doi.org/

$10.19053 / 01227238.11620$

Historia del artículo:

Recibido: 31/07/2020

Evaluado: 24/01/2021

Aprobado: 20/01/2021

Cómo citar este artículo:

Gutiérrez Valderrama, Fredy Mauricioy Leguizamón González, Myriam Cecilia, "Alfabetización Informacional: una vía de acceso a la información confiable" Revista Historia de la Educación Latinoamericana vol.23 no.36 (2021)
La alfabetización mediática e informacional es una dimensión esencial de la educación moral y cívica. También es un derecho fundamental de todos los ciudadanos, en cualquier país del mundo, y por lo tanto, permite a todos proteger su privacidad y encontrar su lugar en una sociedad cuyo entorno tecnológico está cambiando cada vez más rápido.

Audrey Azoulay, directora general de la UNESCO

1 Proyecto de investigación de Maestría en Ambientes Educativos mediados por las TIC, de la Universidad Pedagógica y Tecnológica de Colombia, desarrollado desde el Grupo de Investigación CETIN de la Facultad de Ciencias de la Educación.

2 Magíster en Ambientes Educativos Mediados por las TIC, Universidad Pedagógica y Tecnológica de Colombia, Grupo de Investigación CETIN, reliablefm17@gmail.com

3 Magíster en TIC aplicadas a la Educación. Docente de planta de la Universidad Pedagógica y Tecnológica de Colombia, Grupo de Investigación CETIN, myriam.leguizamon@guptc.edu.co 


\section{Resumen}

Objetivo: Elaborar un plan de acción para implementar la alfabetización informacional ALFIN con los estudiantes del curso TIC y ambientes de aprendizaje de la UPTC, con el propósito de acceder a contenidos digitales confiables, analizando el diagnóstico, el diseño de talleres que permitieran el abordaje de los elementos propuestos.

Originalidad/aporte: Esta discusión presenta una relevancia desde la formación en niveles de educación superior, debido a que la interacción con la información ha venido mutando en los contextos digitales y se hace necesario esclarecer y fortalecer habilidades que lleven a una mirada crítica, de la información a la que se accede.

Método: La investigación fue tomada bajo un enfoque cualitativo, de corte investigación-acción, con una fundamentación teórica.

Estrategia/recolección de información: Se tiene en cuenta que el diseño, la aplicación y análisis de instrumentos de diagnóstico permitieron entender que la universidad cuenta con recursos materiales e intelectuales para el manejo ético de la información.

Conclusiones: La ética es la categoría emergente de esta investigación, su empleo acertado empodera a las sociedades para que construyan conocimiento. Así mismo, la relevancia de está radica en que los docentes de instituciones de educación superior se involucren en su fortalecimiento.

Palabras clave: Alfabetización Informacional; información digital confiable; ética y plan de acción.

\section{Information Literacy: a way of accessing reliable information}

\section{Abstract}

Objective: To elaborate an action plan to implement the information literacy programme (IFLA) with the participation of students of the ICT and learning environments class at the UPTC. The purpose is to access reliable digital content, analyze the diagnosis, and design workshops that allow addressing the proposed elements.

Originality/support: This discussion arises from teaching in higher education. Considering that the interaction with information has mutated due to digital contexts, it is necessary for students to filter and strengthen skills that lead them a critical view of the information they access.

Method: this action research work has a qualitative approach with a theoretical foundation. 
Strategy/ information gathering: the design, application and analysis of diagnostic instruments allowed us to understand that this university has material and intellectual resources for an ethical management of information.

Conclusions: Ethics is the emerging category of this research. This category, when properly used, empowers the construction of knowledge in societies. Likewise, its strengthening depends to a great extent on the involvement of higher education teachers.

Keywords: information literacy; reliable digital information; ethics and action plan.

\section{Alfabetização Informacional: uma via de acesso à informação confiável}

\section{Resumo}

Objetivo: elaborar um plano de ação para implementar a alfabetização informacional ALFIN com os estudantes do curso TIC e ambientes de aprendizagem da UPTC, com o propósito de acessar conteúdos digitais confiáveis, analisando o diagnóstico, o desenho de oficinas que permitiram a abordagem dos elementos propostos.

Originalidade/aporte: esta discussão apresenta relevância a partir da formação nos níveis da educação superior, pois a interação com a informação vem se transformando nos contextos digitais e é necessário esclarecer e fortalecer competências que levam a uma visão crítica da informação que se acessa.

Método: a pesquisa foi tomada sob um enfoque qualitativo, de corte da pesquisa ação, com uma fundamentação teórica.

Estratégia/coleta de dados: tem-se em conta que o desenho, a aplicação e análise de instrumentos de diagnóstico permitiram que a universidade conta com recursos materiais e intelectuais para o manejo ético da informação.

Conclusões: a ética é a categoria emergente desta pesquisa, seu uso correto empodera as sociedades para que construam conhecimento. Assim mesmo, sua relevância reside no fato de que os docentes de instituições superiores se envolvem em seu fortalecimento.

Palavras-chave: Alfabetização Informacional; informação digital confiável; ética e plano de ação. 


\section{Introducción}

A causa de las graves implicaciones que tiene su mal uso, el manejo de la información digital es un tema de preocupación permanente por parte de organizaciones como la UNESCO, que en el año 2011 produjo el documento "Alfabetización mediática informacional: currículo para profesores", a la par que desarrolla talleres en distintos países para hacer ver la magnitud del tema, y moviliza acciones concretas con docentes vinculados directamente con las tecnologías de la información y la comunicación. Para el caso de Colombia, en el año 2018 concertó con docentes encargados de formar licenciados en tecnología e informática y vinculados a la Red de Programas Educativos en Tecnología e Informática de Colombia (REPETIC), a trabajar el tema e intentar definir acciones concretas.

La Alfabetización Informacional (ALFIN) es un concepto transcendental de vieja data, que desde el documento UNESCO hace parte de uno de los dos componentes de la alfabetización: por un lado, lo mediático, y por el otro, lo informacional. Con el transcurrir de los lustros ha empezado a ser notoria la aplicabilidad de su filosofía en los entornos académicos, sin importar la geo-ubicación de los posibles consumidores y prosumidores informacionales. El manejo de la información viene tomando giros impensables, que hacen surgir nuevas preocupaciones por el gran volumen de datos, la mega velocidad con la que se intercambian de forma continua, y la falta de escrúpulos de algunos internautas para publicar información que en ocasiones es manipulada y sesgada para confundir a los usuarios. Usuarios que no siempre están alfabetizados en la forma como acceden, localizan, transforman y comparten esa información.

Consecuentemente, la información seguirá mutando y transformando sociedades que necesitan mantenerse instruidas para tomar oportunas decisiones en la búsqueda incesante de lograr diversos objetivos. He aquí la importancia de alfabetizarse en este sentido. Y de la mano del aspecto ético de la información, las personas que buscan estar actualizadas tendrán la manera de hacer procesos de filtración, contarán con las herramientas que les ayudarán a detectar la desinformación online. Lo primordial de esta labor es que los estudiantes no se convertirán en eslabones de la cadena que masifica falsedades. Por el contrario, estarán calificados para proteger y solidificar la verdad, que es perentorio rescatar y restablecer en función de una humanidad equitativa, dinámica y floreciente.

Ahora bien, con base en esta pretensión se desarrolló la investigación, la cual da cuenta del trabajo realizado, en el que se idearon cuatro talleres tipo total $1^{4}$, con actividades cognitivas y procedimentales para apoyar las competencias en los siete elementos de la Alfabetización Informacional (ALFIN), propuestos por la UNESCO 5 . Adicionalmente, para la evaluación de estos talleres se mantuvieron en mente indicadores de competencias en cada elemento que define el uso particular de los mismos. En consecuencia, se diseña un plan de acción con base en el diagnóstico recogido de las entrevistas realizadas a: funcionario de biblioteca, abogado jurídico, docente de TIC de la UPTC; sobre la temática de Alfabetización Informacional. Al

4 María Chisaba, César Hernández y Marisol Ramírez, "El taller como estrategia metodológica para el aprendizaje de expresiones idiomáticas del inglés en niños de cuarto de primaria" (tesis de grado de licenciatura en Educación Básica con énfasis en Humanidades, Universidad Libre, 2013).

5 Carolyn Wilson et al., Alfabetización Mediática e Informacional: Currículum para profesores. París: UNESCO, 2011. 
igual, se tienen en cuenta los resultados de aplicación de talleres, realizados en el curso TIC, en forma de prueba piloto. El plan busca apoyar al docente para que los estudiantes efectivamente aprendan de los elementos ALFIN y empiecen a apropiarse de esas competencias desde el curso de TIC y ambientes de aprendizaje.

\section{Referentes teóricos}

\section{Antecedentes y estado del arte}

A continuación se presenta una revisión de los últimos diez años, relacionada con el tema de la alfabetización informacional, se destacan investigaciones producto de la revisión de revistas indexadas en los ámbitos colombiano e iberoamericano, resaltando algunos referentes como: Revista Iberoamericana de Educación Superior de la Universidad Nacional Autónoma de México y Universidad de México; Revista de Estudios Sociales de la Universidad de los Andes en Colombia; Revista Historia de la Educación Latinoamericana de la UPTC de Colombia; Revista Letral de la Universidad de Granada, España; Comunicación y Medios de la Universidad de Chile; Revista española de Documentación Científica de España.

La investigación cuantitativa correlacional "Factores personales que inciden en la autovaloración de futuros maestros sobre la dimensión pedagógica del uso de TIC", resalta que la competencia digital contiene una dimensión llamada alfabetización informacional que, "implica saber plantear estrategias y procesos de resolución de problemas relacionados fundamentalmente con la información" ${ }^{\text {. }}$. Los resultados proyectan que la población muestra pertenecientes a carreras de pedagogía de una universidad chilena de carácter público, estudiantes que sienten contar con la competencia digital para el uso pedagógico de las TIC con la premisa del corte tradicionalista. Para esta iniciativa se mantuvieron en mente perspectivas de edad, género, nivel educativo de formación inicial docente y conectividad.

La investigación: Desarrollo de la competencia mediática en el contexto iberoamericano, en el que se exponen qué tanto la digitalización como la interactividad "favorecen la inmediata difusión de contenidos audiovisuales"7 a través de distintos dispositivos que son "propios de la sociedad hipermedia"8; al instante tienen en cuenta los informes presentados por la UNESCO y por la ONU, en donde la investigación tiene el objetivo de "saber cuáles son las diferencias según las seis dimensiones que componen la competencia mediática en niños de primaria, jóvenes de secundaria, estudiantes universitarios y docentes no universitarios"9. Para este fin ejecutaron cuatro cuestionarios, creando indicadores sociales con el objetivo

6 Carolina Flores Lueg y Rosabel Roig Vila. "Factores personales que inciden en la autovaloración de futuros maestros sobre la dimensión pedagógica del uso de TIC", Revista RIES, vol. 10, n. ㄱ 27, 2019.

7 Ignacio Aguaded, Isidro Marín y Mari Carmen Caldeiro "Desarrollo de la competencia mediática en el contexto iberoamericano", Revista Letral, n. ${ }^{\circ} 20,2018$.

8 Ibíd.

9 Joan Ferrés, "La competencia en comunicación audiovisual: dimensiones e indicadores", Comunicar, vol. 29, n. ${ }^{\circ}$ 15, 2007. 
de determinar las seis dimensiones y el posterior análisis de los datos. En consecuencia, y buscando una aproximación a la definición de la ALFIN, de la que se dice "empodera a las nuevas generaciones porque, si aprenden a evaluar la confiabilidad informativa, aprenden también a tomar decisiones sobre la base de la información pensada"10. En esa perspectiva, este concepto puede contribuir a que, desde la mirada ética, la cual es parte estructural en este trabajo, se cuente con los elementos ALFIN para prevenir la desinformación online.

La investigación documental, llamada "Estado de la cuestión de la formación docente en educación en medios", propuso hacer el análisis de 28 artículos en relación con la formación docente en los medios, y, deteniéndose en cuáles son los elementos representativos de estos, se encontró que coinciden en que "la formación en educación en medios para los (futuros) profesores es clave para contar con una ciudadanía preparada para la era digital"11.

Otro abordaje se presenta con el proyecto que planteó como objetivo "ofrecer una sistematización y organización de las lecciones aprendidas desde los programas más representativos de Alfabetización Informacional (ALFIN) en universidades iberoamericanas" ${ }^{\prime 2}$, teniendo como método la "aplicación de un modelo de captura de lecciones aprendidas basado en la triangulación de diferentes fuentes de información y métodos de investigación". Lo cual les permitió mostrar esos 75 resultados, que hacen parte de cuatro componentes que serán un faro para ser aplicados en los programas de formación de la ALFIN.

Otra investigación ${ }^{13}$ centra su atención en las distintas competencias con las que se debe contar al interactuar con la información y además las "destrezas prácticas necesarias para actuar con autonomía en diversos contextos, para relacionarse y comunicarse eficazmente y para participar y cooperar socialmente"14 y cómo en investigaciones realizadas se destaca la relación cada vez más cercana de los estudiantes con la información, dada la exposición a los distintos dispositivos tecnológicos y estos a las TIC. En consecuencia, se resalta que el desarrollo de competencias tecnológicas y mediáticas va empoderado por la adquisición de habilidades que mejoren la interacción con la tecnología de una manera crítica y autónoma.

Otro estudio que marcó pautas para el desarrollo de la investigación fue el llevado a cabo para buscar el significado no solo de la alfabetización digital sino audiovisual, con el fin de llegar a una conceptualización de la competencia mediática en la que confluyen aspectos como los destinatarios, la conceptualización que se utiliza en cada uno de ellos, el tipo de taxonomía, las propuestas didácticas e indicadores. Esto les permitió a las autoras "afirmar la necesidad de la convergencia termino-lógica, así como de la elaboración de recursos, a partir de los indicadores definidos, que incidan en los distintos ámbitos de la competencia

10 Roxana Morduchowicz, Ruidos en la web. Cómo se informan los adolescentes en la era digital. Buenos Aires: Ediciones B, 2018.

11 Pablo Andrada "Estado de la cuestión de la formación docente en educación en medios", Comunicación y Medios, n. - 31, 2015.

12 Alejandro Tirado y María Pinto, "75 lecciones aprendidas en programas de alfabetización informacional en universidades iberoamericanas", Revista española de Documentación Científica, vol. 37, n. 3, 2014.

13 Concepción Naval y Elena Arbués, "La alfabetización mediática de los futuros profesores de educación primaria y secundaria", en Sociedad del conocimiento y educación, coord. Lorenzo García. Madrid: Universidad Nacional de Educación a Distancia, 2012.

14 Partnership for 21st century skills (P21), "Framework definitions", 2009. 
mediática de una manera efectiva y sirvan para llevar a cabo actuaciones didácticas en los distintos grupos que componen la sociedad actual"15.

En suma, se logra definir que los proyectos anteriores se realizaron en contextos universitarios, donde se incorpora el concepto Alfabetización Informacional en procura de ser efectivos a la hora de buscar, evaluar, compartir y organizar la información. Y son guiados por docentes y personal bibliotecario, quienes poseen un conocimiento sólido relacionado con la actividad informacional.

Figura 1. Antecedentes más representativos en el tema de la investigación

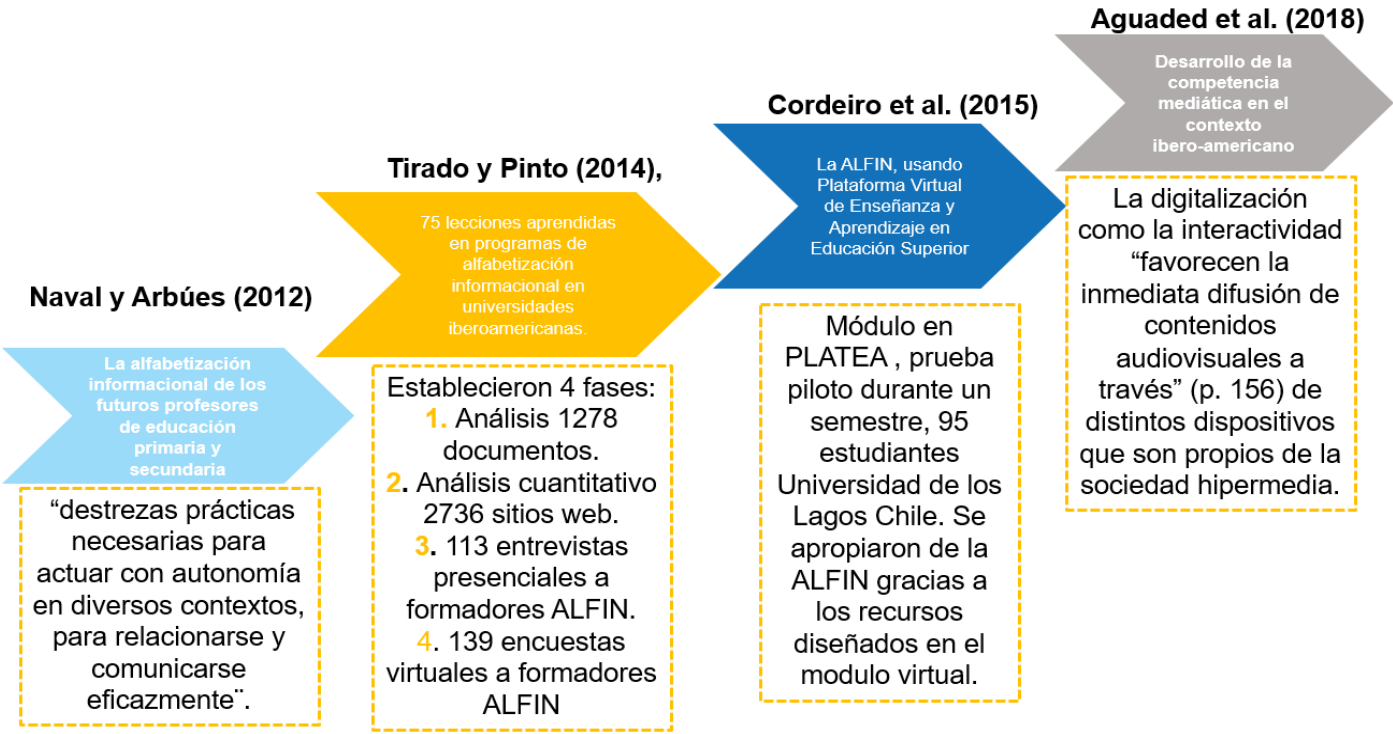

Fuente: Fredy Gutiérrez, tesis de maestría en Ambientes Educativos Mediados por TIC, 2020.

\section{Alfabetización mediática y alfabetización informacional}

El uso cada vez mayor de las tecnologías de la información, en la vía de la denominada convergencia digital — que algunos consideran una moda-, hace que, quienes se deciden por la innovación tecnológica declaren que es actualmente una necesidad y forma parte de la cotidianidad de los usuarios que hacen (o hacemos) parte de la sociedad de la información y el conocimiento ${ }^{16}$. En este sentido, esta cotidianidad no implica desconocimiento de aspectos legales que se desprenden de aspectos éticos y de abordar como un tema curricular lo pertinente.

15 M. ${ }^{a}$ Amor Pérez y Águeda Delgado, "De la competencia digital y audiovisual a la competencia mediática: dimensiones e indicadores", Comunicar, vol. XX, n. ${ }^{\circ}$ 39, 2012.

16 Diana Elvira Soto Arango, Fredy Yesid Mesa Jiménez y Edgar Orlando Caro, "Convergencia digital en la universidad colombiana. Del siglo XX al XXI", Revista Historia de la Educación Latinoamericana, vol. 14, n. ${ }^{\circ}$ 19, 2012. 
En consecuencia, al adentrarnos en el tema objeto de estudio, es necesario iniciar contextualizando a los lectores de los conceptos de alfabetización mediática e informacional. A pesar de que la alfabetización mediática e informativa parecen ser dos campos independientes, ambos conceptos comparten el objetivo común de cultivar la habilidad de los individuos para acceder, comprender, utilizar y crear mensajes mediáticos o informativos, y en el área de la alfabetización siempre han aparecido estrechamente relacionados ${ }^{17}$. Como principal diferencia,

(...) la alfabetización mediática posee un conocimiento académico arraigado en el estudio de los medios y de las ciencias sociales, y se dirige al estudio de aspectos clave del fenómeno del medio de masa, como son los mensajes mediáticos, las industrias y audiencias, así como el efecto de los medios, lo cual provoca que la alfabetización mediática adopte enfoques más analíticos. ${ }^{18}$

La alfabetización informacional se concentra en el análisis de información y, por tanto, se centra fundamentalmente en el análisis textual y en el valor investigador del hallazgo de la verdad en los documentos. Esto tiene que ver con el análisis crítico de la calidad de la información, pero no examina al público ni los efectos de dicha información ${ }^{19}$.

Articulando la alfabetización informacional (ALFIN), esta "enfatiza la importancia del acceso a la información, la evaluación y el uso ético de dicha información"20; de igual manera, la ALFIN es concebida como parte de las habilidades que las personas deben adquirir para ser competentes, aspectos estos relacionados con el nivel de capacidad que tienen para "• reconocer sus necesidades de información; • localizar y evaluar la calidad de la información; • guardar y recuperar información; • usar la información de una forma eficaz y ética; • aplicar la información para crear y comunicar conocimientos"21.

En la Universidad Javeriana se documenta la investigación titulada "Recolectores, verificadores y reflexivos: perfiles de la competencia informacional en estudiantes universitarios de primer semestre"22 de las universidades Javeriana e Industrial de Santander (UIS), trabajo que se centró en explorar aspectos relacionados con esta competencia, orientando el concepto desde lo cultural y social para referirse a una práctica que se caracteriza por ser diferenciada, en contexto y frecuencia, en el que los autores analizaron 285 educandos de las dos universidad objeto de estudio, usando como técnicas e instrumentos para colectar los datos un test de perfil, entrevistas y el reporte de protocolos verbales. En sus conclusiones afirman: "Estos perfiles no son fijos, sino más bien fluidos, y se concretan de manera diferente según la tarea académica. Se recomienda realizar estudios de perfiles informacionales en estudiantes tanto

17 Alice Lee y Clement So, "Alfabetización mediática y alfabetización informacional: similitudes y diferencias" Comunicar, vol. 21, n. ${ }^{\circ}$ 42, 2014.

18 Ibíd., 144.

19 Jesús Lau, "Conceptual Relationship of Information Literacy and Media Literacy", en Conceptual Relationship of Information Literacy and Media Literacy in Knowledge Societies, París: UNESCO, 2013.

20 Wilson et al., Alfabetización mediática.

21 Ibíd., 42.

22 Harold Castañeda-Peña et al., "Recolectores, verificadores y reflexivos: perfiles de la competencia informacional en estudiantes universitarios de primer semestre", Revista Interamericana de Bibliotecnología, vol. 33, n. ${ }^{0}$ 1, 2010. 
de secundaria como universitarios, para diseñar políticas educativas sobre competencia informacional"23.

Estas investigaciones convergen en el tema central de esta investigación y aportaron elementos destacados para tener en cuenta y para reorientar, tanto la pesquisa y revisión teórica como aspectos metodológicos.

\section{Elementos de la Alfabetización Informacional}

Es fundamental mencionar cada uno de los siete elementos ALFIN ${ }^{24}$ presentados en la figura 2.

1. Definir y articular necesidades de información. Hace referencia a la planificación de la información que se precisa y en qué instante hay esa necesidad informativa.

2. Localizar y evaluar información. Su meta es ubicar y cómo llegar acertadamente a esa información.

3. Evaluar la información. Está ligada al hecho de revisar la veracidad, actualidad y objetividad informacional.

4. Organizar la información. Su objetivo es estructurar de forma coherente los datos con relación a los propósitos para generar productividad informativa.

5. Uso ético de la información. Hacer un uso responsable de la información.

6. Comunicar información. Busca exponer la información, teniendo presente al público, utilizando además los formatos adecuados para ese principio.

7. Uso del conocimiento de las TIC para procesar la información. Aprecia lo relacionado con las herramientas tecnológicas para gestionar de manera efectiva la información.

23 Ibíd., 42.

24 Francisco J. Sáenz, "Alfabetización Informacional: qué es y cómo se adquiere", InVito, el blog de la Biblioteca Francisco de Vitoria: https://diarium.usal.es/vito/2013/10/02/alfabetizacion-informacional-que-es-y-como-seadquiere/. 


\section{Figura 2. Elementos de la Alfabetización Informacional}

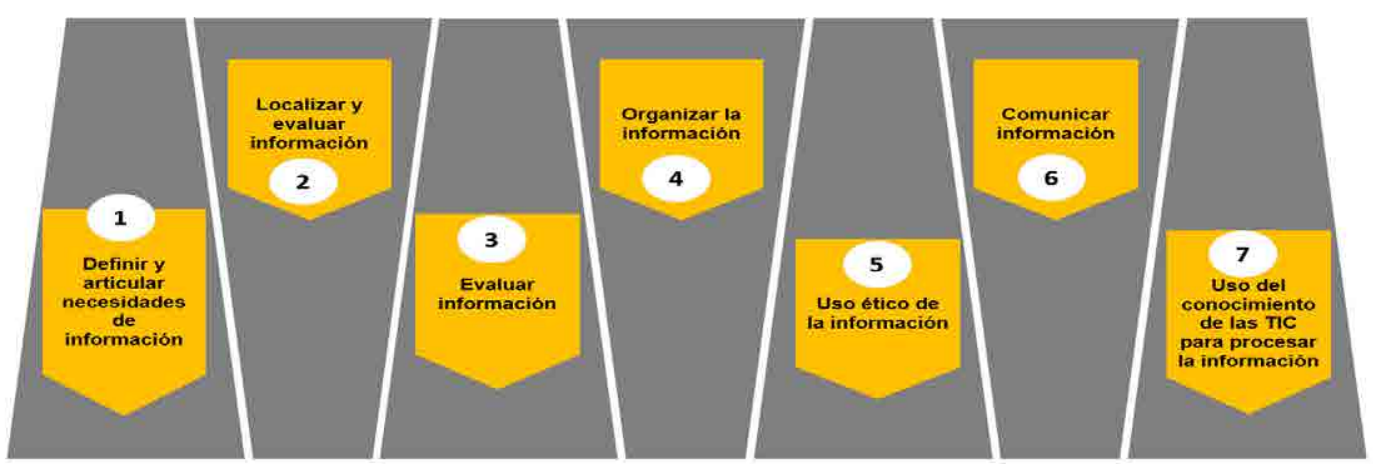

Fuente: Fredy Gutiérrez, con base en Carolyn Wilson et al., Alfabetización mediática, 8.

\section{Contenido digital}

Cuando se habla de información, se pueden tener distintas visiones desde los campos de formación o contextos en los que se movilizan los términos; para el caso de esta investigación se aborda la alfabetización informacional, en el entendido de que la información se materializa en contenido impreso o digital, y en este proyecto se asume el contenido digital como "información digitalizada, desarrollada o adquirida con el objetivo de ser accesible e intercambiable para favorecer el diálogo cultural y el desarrollo económico de los usuarios de esta tecnología"25.

Estos contenidos deben contribuir a:

- Impulsar el uso y el acceso de todos los usuarios de la internet con la finalidad de apoyar su desarrollo profesional, social y cultural.

- Asegurar la utilización del potencial máximo de los contenidos digitales por parte de los usuarios.

- Crear condiciones favorables para aumentar la distribución y el uso de contenidos digitales en la internet, adaptando de la mejor manera posible los aspectos culturales y lingüísticos de sus usuarios.

Los contenidos digitales de la mano de las TIC han permitido la masificación y circulación de la información. "La utilización del código digital como sistema para almacenar o transmitir todo tipo de información, sean textos escritos, palabras, música, números o imágenes,

25 Enrique Ruiz Velasco, "Desarrollo de contenidos digitales educativos en comunidades de aprendizaje" http:// bibliotecadigital.conevyt.org.mx/colecciones/documentos/somece2002/Grupo2/Ruiz.pdf 
comporta una revolución en la organización de todas las actividades relacionadas con la difusión de la información y el conocimiento"26.

\section{Competencias digitales en los docentes}

Las competencias digitales, al ser afines con las capacidades de integración e interacción que el docente, o futuro docente, debe tener con las herramientas TIC, merecen ser presentadas en este documento, particularmente por las expectativas que, en cuanto a las prácticas docentes, presenta el Ministerio de Educación Nacional colombiano en el libro Competencias TIC para el desarrollo profesional docente, del que provienen las siguientes definiciones:

- Competencia pedagógica: Capacidad de utilizar las TIC para fortalecer los procesos de enseñanza y aprendizaje, reconociendo alcances y limitaciones de la incorporación de estas tecnologías en la formación integral de los estudiantes y en su propio desarrollo profesional.

- Competencia comunicativa: Capacidad para expresarse, establecer contacto y relacionarse en espacios virtuales y audiovisuales a través de diversos medios y con el manejo de múltiples lenguajes, de manera sincrónica y asincrónica.

- Competencia investigativa: Capacidad de utilizar las TIC para la transformación del saber y la generación de nuevos conocimientos. ${ }^{27}$

Esta última, corresponde con la interacción que se da con la información y esto a su vez con la alfabetización informacional. La revisión y contraste con la alfabetización informacional se muestra en tanto que esta investigación se enmarca en una asignatura del componente interdisciplinar en la Facultad de Ciencias de la Educación, como componente fundamental en la formación de docentes.

\section{Proceso metodológico}

Con el ánimo de dar respuesta a cuáles son los aspectos por considerar para la elaboración de un plan de acción para acceder a contenido digital confiable, esta investigación se suma a la preocupación de otros investigadores que han indagado sobre la carencia de rigurosidad, confiabilidad y credibilidad de los contenidos disponibles en la red, por el acceso sin control, el anonimato y la falta de regulación para publicar en Internet. Lo que genera más desinformación que la construcción de una inteligencia colectiva ${ }^{28}$.

26 Pablo Lara, "Uso de contenidos digitales: tecnologías de la información, sociedad del conocimiento y universidad", Revista de Universidad y Sociedad de Conocimiento, vol. 2, n. ${ }^{\circ} 2,2005$.

27 Ministerio de Educación Nacional, Competencias TIC para el desarrollo profesional docente. Bogotá: Imprenta Nacional, 2013.

28 Francisco Gómez, "Desinformación en Internet y hegemonía en redes sociales", Gestión de las Personas y Tecnología, vol. 5, n.16, 2013. 
A escala global hay una preocupación, y no es ajena al contexto colombiano, relacionada con la vasta cantidad de información que se encuentra en diversos escenarios online y la complicación que implica, para algunas personas, detectar qué tanta de esa información ha sido malintencionadamente sesgada o alterada para confundir y manipular.

En cuanto al entorno colombiano, la alfabetización informacional se sigue observando bajo la perspectiva de la brecha digital centrada en cuestiones de infraestructura y relegando el aspecto informacional, que es el que se procura potenciar en esta investigación.

En definitiva, esta investigación pretende dejar los insumos ALFIN suficientes para que los participantes de este proyecto estén en capacidad de resaltar la verdad a la hora de definir, evaluar y comunicar información de forma ética.

Esta problemática conllevó el desarrollo de un plan de acción que permitiera implementar la ALFIN con los estudiantes del curso TIC y ambientes de aprendizaje, con el propósito de acceder a contenido digital confiable. Para esta iniciativa se diseñaron y ajustaron instrumentos de recolección de información para cada una de las etapas de la investigación-acción, puesto que para el tema de la alfabetización mediática e informacional se encuentran ya diseñados y validados algunos instrumentos que, para el caso particular, precisó utilizar una encuesta diagnóstica utilizando el formato Google forms a la población muestra. También se realizaron tres entrevistas a personal institucional objeto de estudio. Además, se plantearon cuatro talleres con sus respectivas matrices de evaluación. En consecuencia, estos instrumentos se articularon con los objetivos específicos, como se presenta a continuación: las fases propuestas por Lewin ${ }^{29}$

\begin{tabular}{|c|c|c|}
\hline Objetivo especifico & $\begin{array}{c}\text { Fase de la investigación- } \\
\text { acción, según Lewin }\end{array}$ & Instrumentos \\
\hline $\begin{array}{c}\text { Diagnosticar la población muestra en } \\
\text { cuanto al acceso, búsqueda y selección } \\
\text { de la información en relación con las } \\
\text { competencias de la ALFIN con contenidos } \\
\text { digitales }\end{array}$ & $\begin{array}{c}\text { Esclarecer y diagnosticar la } \\
\text { situación problemática }\end{array}$ & $\begin{array}{c}\text { Encuesta diagnóstica. } \\
\text { Entrevistas }\end{array}$ \\
\hline $\begin{array}{c}\text { Diseñar talleres prácticos con contenidos } \\
\text { digitales publicados en la web, que } \\
\text { permitan abordar los elementos de la } \\
\text { ALFIN }\end{array}$ & $\begin{array}{c}\text { Fomentar estrategias de } \\
\text { acción para solucionar } \\
\text { el problema. } \\
\text { Aplicar y evaluar las } \\
\text { estrategias de acción }\end{array}$ & Matriz de evaluación \\
\hline
\end{tabular}

29 Kurt Lewin, "Acción-investigación y problemas de las minorías", Revista de Psicología Social, vol. 3, n. 2, 1988. 


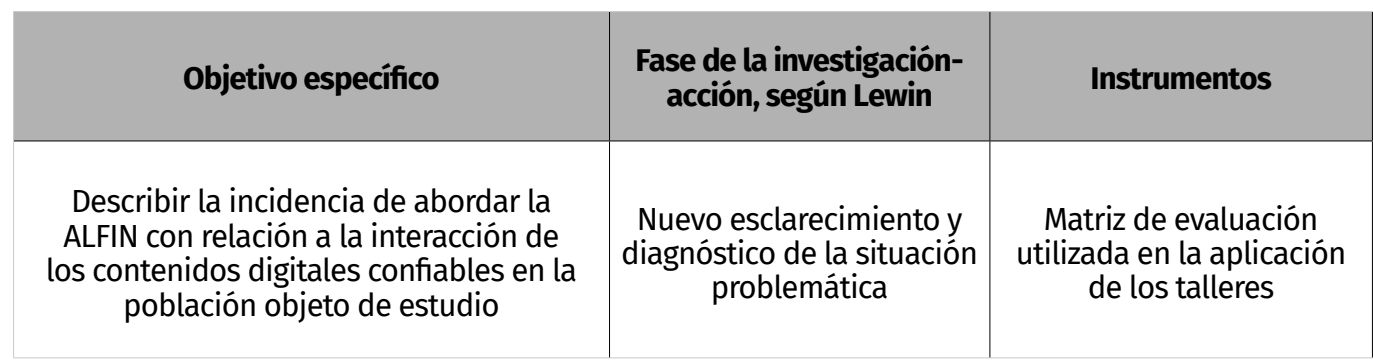

Fuente: Elaboración propia.

La muestra está compuesta por dieciséis estudiantes de primer semestre de las licenciaturas en educación de la Universidad Pedagógica y Tecnológica de Colombia, esto en relación con la asignatura de TIC y ambientes de aprendizaje, del II semestre de 2019, con quienes se desarrolla el diagnóstico y la prueba piloto. Esta asignatura corresponde al área interdisciplinar de la Facultad de Ciencias de la Educación, y es el espacio académico que permite hacer efectivo el diálogo de saberes desde las distintas disciplinas que hacen parte de la Facultad, pues este curso se programa en horario de franjas académicas, y los estudiantes se inscriben al grupo que se ajusta a sus tiempos.

En cuanto a las categorías de análisis, se tomaron los elementos dados por el documento de la UNESCO, referenciados en la figura 2 de este documento, así como los indicadores allí definidos. Esto en virtud de la validación que tiene el instrumento y que se ajusta a lo que se quiere valorar.

Para la fase de análisis de datos se acudió principalmente a la triangulación entendida como "la combinación de dos o más teorías, fuentes de datos, métodos de investigación, en el estudio de un fenómeno singular". Y para el presente trabajo la triangulación de datos es la más acertada, además que "se refiere a la confrontación de diferentes fuentes de datos en los estudios y se produce cuando existe concordancia o discrepancia entre estas fuentes"30, el procesamiento de los datos se realiza en el software Atlas.ti.

Adicionalmente, fue necesario acudir a la estadística, y lo descriptivo se manejó en el programa Excel, para dar mayor apertura a la lectura de los resultados, por lo cual esta se utiliza

...como un conjunto de métodos, técnicas y procedimientos para el manejo de datos, su ordenación, presentación, descripción, análisis e interpretación, que contribuyen al estudio científico de los problemas planteados en el ámbito de la educación y a la adquisición de conocimiento sobre las realidades educativas. ${ }^{31}$

\section{Fases de la investigación}

La primera fase denominada "Esclarecer y diagnosticar la situación problemática" - objeto de estudio, abordó las siguientes acciones en concreto: Diseño y formulación de instrumentos,

30 Norman Denzin, ed., Sociological Methods. A Sourcebook, Chicago: Aldine Publishers, 1970.

31 Javier Gil, "La estadística en la investigación educativa", Revista de Investigación Educativa, vol. 21, n. ${ }^{\circ}$ 1, 2003. 
aplicación de instrumentos, triangulación de instrumentos con los elementos de la ALFIN, esclarecer la situación problema.

Este momento permitió ejecutarse apoyado entre otras fuentes e instrumentos de la encuesta aplicada a la población muestra, donde se identificó que hay unas fortalezas y debilidades con relación a los elementos ALFIN que se deben potenciar para que el uso informacional sea acorde a las necesidades actuales de saturación y manipulación informacional. En la siguiente figura se presentan los resultados del instrumento, se resalta la emergencia del componente ético, de todos los indicadores de las categorías de la alfabetización informacional.

\section{Figura 3. Síntesis del diagnóstico sobre elementos de la ALFIN}

\section{Sintesis del diagnostico sobre elementos de la ALFIN}

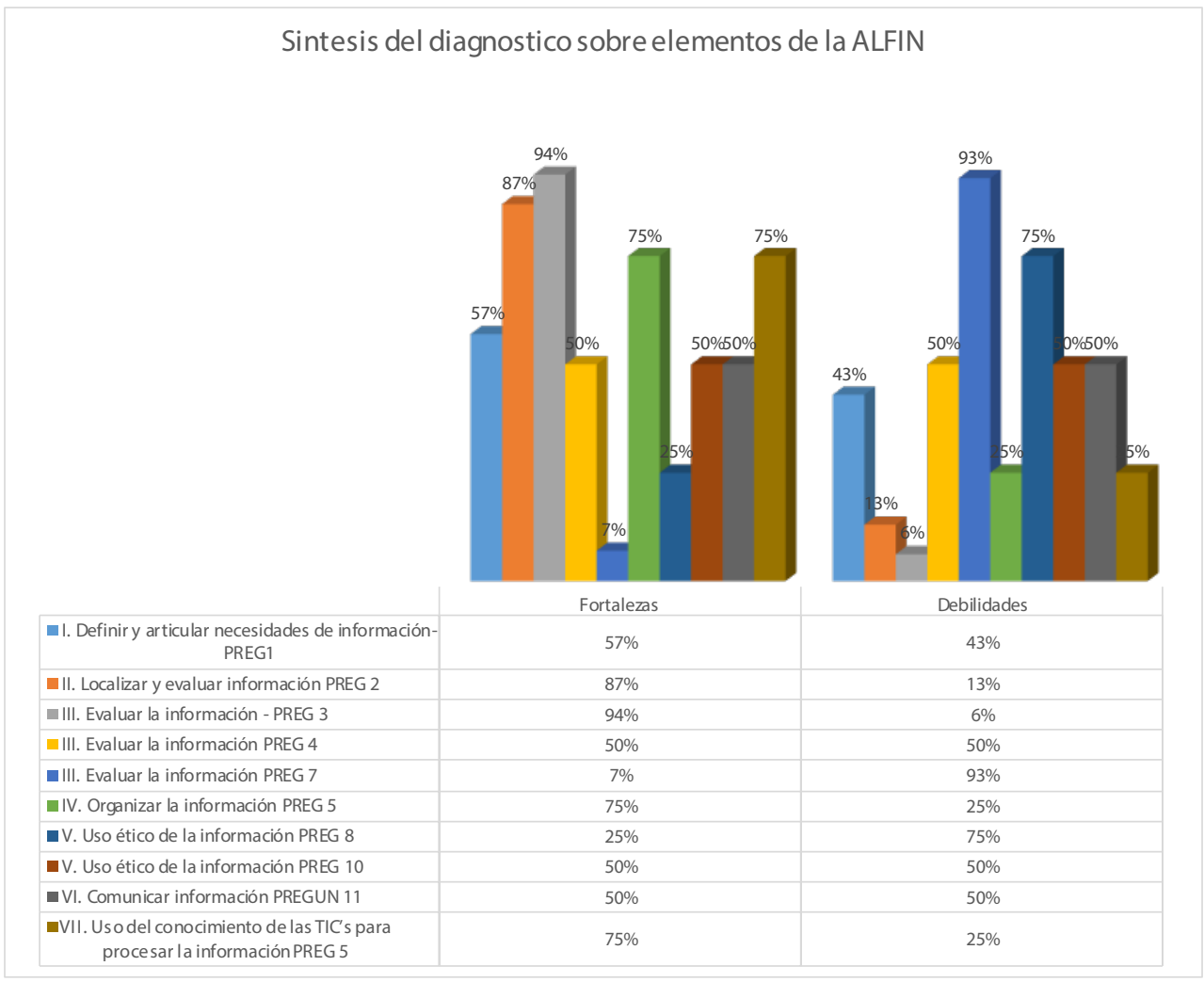

Fuente: Elaboración propia.

Así mismo, se hace uso como instrumento de las entrevistas realizadas al abogado de la oficina jurídica, al bibliotecólogo a cargo de la capacitación en bases de datos e información digital y docente del área TIC y ambiente de aprendizaje de la UPTC. De la revisión de la información recogida de esta triada de fuentes primarias se establece como primer insumo que hay valiosos elementos humanos y económicos ligados al manejo ético de la información. Que en esta alfabetización sería significativa una asignatura de pregrado, articulada con el objetivo de dar herramientas a los estudiantes en la búsqueda, organización, evaluación y uso del conocimiento TIC de la información. No obstante, los actores mencionados aducen que 
algunos estudiantes carecen de información precisa a la hora puntual de ejercer el aspecto ético en sus procederes académicos.

La información recogida fue procesada en el software Atlas.ti, mediante el cual se visibilizaron los siguientes términos como los de mayor representatividad: plagio, propiedad intelectual, estilos de citación y gestores bibliográficos. Lo anterior dio pistas para empezar la segunda fase de la investigación, fortaleciendo sobre todo aquellos elementos prioritarios para los participantes de la investigación.

\section{Figura 4. Nube de palabras a partir del análisis de las entrevistas realizadas a funcionarios y docentes de la UPTC relacionados con el manejo digital y ético de la información}

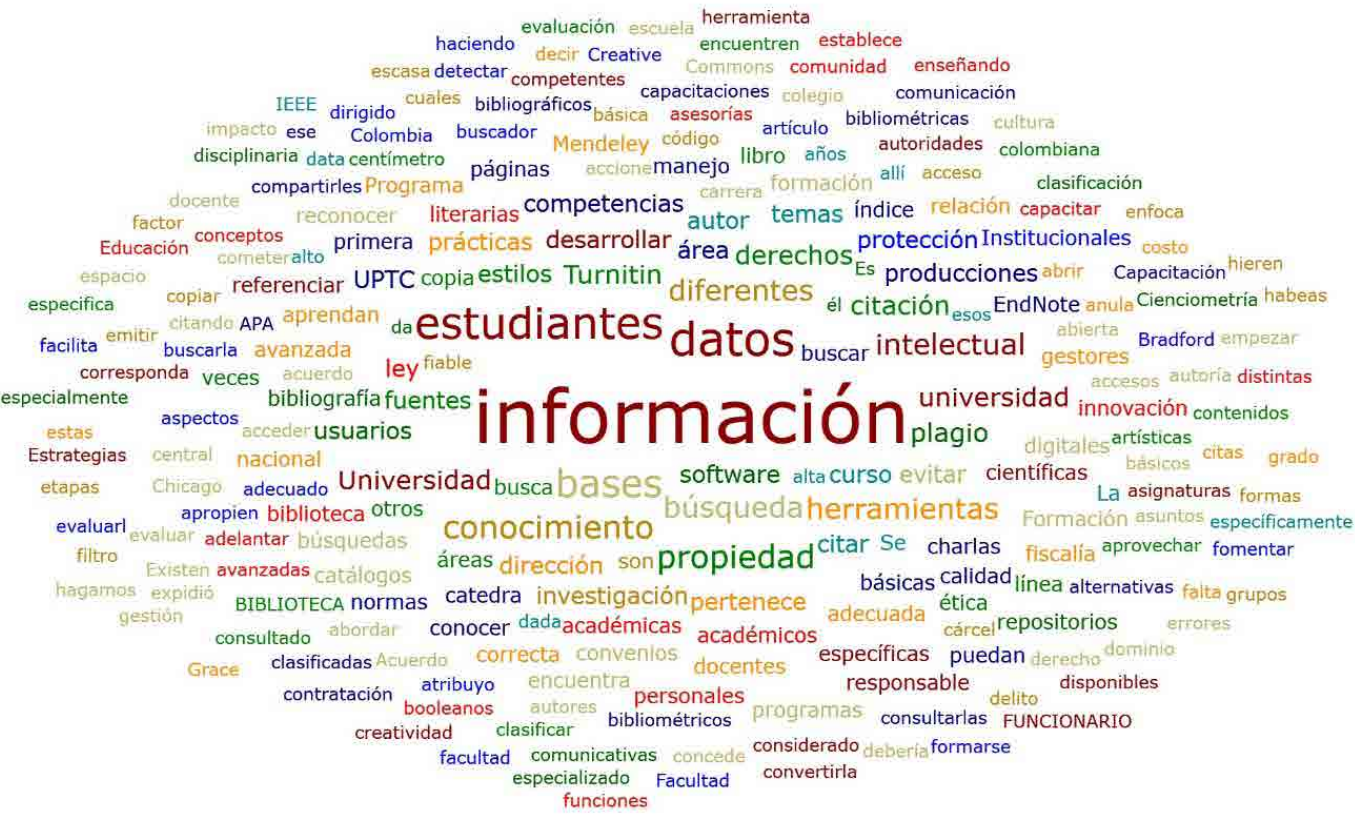

Fuente: Elaboración propia. 
Figura 5. Red semántica del análisis a las entrevistas realizadas al personal de la UPTC

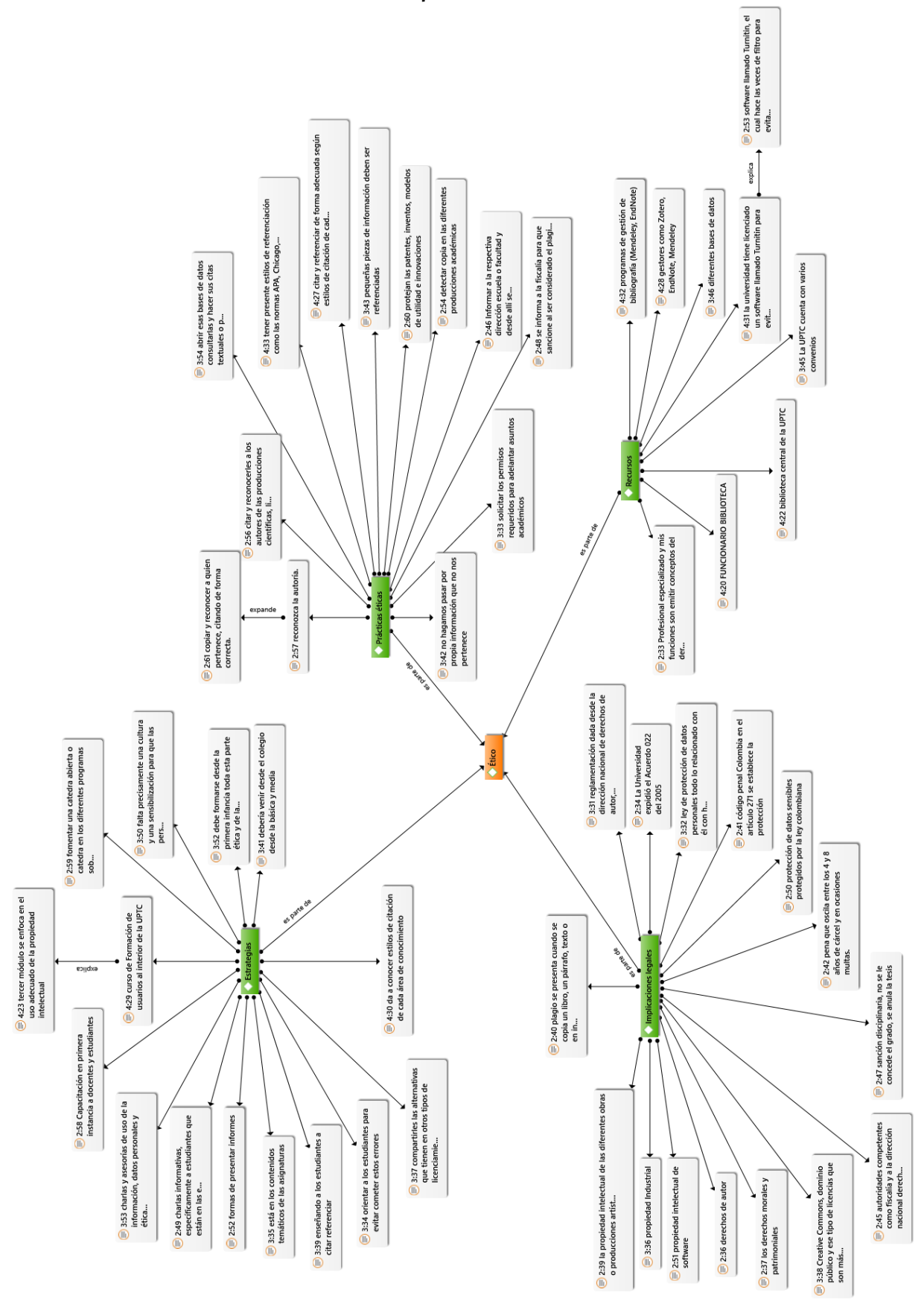

Fuente: Elaboración propia. 
A raíz de la revisión de las fuentes abordadas fue posible esclarecer los aspectos que rodean el problema, los cuales se organizan de manera jerárquica. Es así como, a nivel institucional, desde la percepción de la oficina jurídica con el abogado que orienta lo relacionado con derechos de autor, y desde la mirada del funcionario de la biblioteca encargado de procesos de formación en manejo de bases de datos e información digital, se analiza que la institución cuenta con recursos humanos y económicos para capacitar, informar y transformar la información con la que los estudiantes lidian permanentemente.

Por su parte, a nivel de Facultad, con el docente y los estudiantes que participaron en la investigación, se observa que en el curso de TIC y ambientes de aprendizaje se encuentra lo relacionado con el acceso a bases de datos. Sin embargo, se requiere hacer mayor énfasis en la Alfabetización Informacional. Los estudiantes muchas veces acceden a información no confiable porque han sido las prácticas que han apropiado; desconocen, en algunos casos, cómo acceder a información confiable o los riesgos de referenciar algunas fuentes. En consecuencia, es urgente seguir trabajando para consolidar la apropiación y puesta en marcha de los elementos ALFIN. En este caso, los resultados mostraron mayor necesidad en cuatro de los siete elementos, así: definir y articular necesidades de información, evaluar la información, uso ético y comunicar información, como se presentó en la figura 3.

La segunda fase, denominada "Fomentar estrategias de acción para solucionar el problema", implicó el diseño del plan de trabajo, diseño de talleres y diseño de matriz de evaluación para trabajo de campo. El eje central de este momento se orientó al diseño de cuatro talleres, con la premisa de reforzar los elementos ALFIN que en la fase diagnóstica se mostraron menos favorecidos y articulados a los contenidos programáticos trabajados por el docente del área TIC y ambientes de aprendizaje.

Como complemento, se revisaron los contenidos programáticos del área intervenida para organizar un plan de trabajo que contara con un cronograma que determinara fecha de diseño, implementación y análisis. Se revisaron los componentes teóricos y las herramientas TIC para el desarrollo de los talleres. En cuanto a la estructura y la temática, se examinaron los aspectos que lograran abordar el desarrollo de alguna actividad relacionada con los temas de la materia, pero con acciones direccionadas a la ALFIN. En consecuencia, para efectos de la valoración, se diseñó una matriz que permitiera evaluar cada taller de manera cualitativa con la escala diseñada, desde los cuatro elementos ALFIN a reforzar, con sus respectivos indicadores, para realizar un seguimiento que ilustrara el fortalecimiento de los elementos ALFIN.

La estructura de los talleres constaba, para esta etapa, de una actividad cognitiva que busca dar las bases conceptuales sobre el tema a estudiar, apalancada en hipertexto y multimedia, de manera que los estudiantes exploren la variedad de recursos a su disposición para ampliar sus capacidades en términos de información. El taller incorpora, así mismo, una actividad procedimental para que la población muestra ejercite y fortalezca los elementos ALFIN.

A manera de ilustración, en lo que respecta al taller n. ${ }^{\circ} 1$, los contenidos programáticos del curso fueron derechos de autor y netiqueta. Mediante formato Google forms se les explica la dinámica de la actividad y el tiempo con el que cuentan para proceder. También, se les informó a los estudiantes la estructura para referenciar algunas fuentes de información como: blogs, artículos de revistas online y páginas web (contenido digital). Al momento de 
hacer la referencia tenían dificultades en el uso del orden de la estructura para referenciar en normas APA versión 6. Al citar las referencias, algunos de ellos copiaban solamente la URL del sitio. Los demás referenciaban con fallas en el manejo del autor. Se observa que algunos desconocían el manejo de las referencias para una página web. Ahora bien, la matriz utilizada de evaluación permitió determinar que el promedio del grupo en la escala cuantitativa era aceptable.

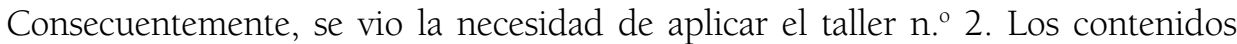
programáticos fueron: redes sociales y adicción a Internet. Se les informó a los estudiantes que debían realizar referencias bibliográficas de páginas web, artículos, tesis, blogs. Adicionalmente, referenciar videos online y podcast. En términos generales, la población reflejó una aplicabilidad importante en relación con el tema de normas APA; la falla fue la referenciación de los videos online y el podcast. La matriz de evaluación los ubicó de nuevo en aceptable. Fue necesario aplicar otro taller para subsanar los errores.

Para el taller n. ${ }^{\circ} 3$ los contenidos fueron "ciber dependencia" y "ciber acoso", en el cual los estudiantes debían referenciar un video del Ministerio de las Tecnologías e Información en TIC sobre ciber acoso e identificar las características de cinco buscadores online como Dialnet, Scielo, Google académico, Redalyc. La mayoría de los estudiantes comprendieron de forma adecuada la sintaxis para referenciar un video en línea con formato APA. Además, reflejaron un alto grado de satisfacción en concordancia con los cinco mejores buscadores de información académica online. En este punto, haciendo uso de la matriz de evaluación, la escala los ubicó en bueno, lo cual se plasma en el mejoramiento por parte de los estudiantes con relación a la búsqueda, evaluación y referenciación de la información de forma ética.

Resumiendo, es importante hacer una comparación entre las fortalezas y debilidades de los elementos ALFIN entre el diagnóstico y la aplicación de los talleres. Este arrojó que, entre los cuatro elementos trabajados que presentaban dificultades, se produjo una reducción gradual e importante, y son: elemento n. ${ }^{\circ} 1$, definir y articular necesidades de información (disminución del 43 al $20 \%$ ); elemento n. ${ }^{\circ}$ 3, evaluar la información (reducción del 50 al 20 \%); elemento n. ${ }^{\circ}$ 5, uso ético de la información (descenso del 63 al 27 \%), el más relevante y eje central de este trabajo y en el que se ve una mejoría sustancial. Por último, en el elemento n. ${ }^{\circ}$ 6, comunicar la información, hay una contracción del 50 al 20 \%. Lo descrito permite deducir que la aplicación de esta estrategia generó un cambio positivo, una transformación significativa que empodera a los participantes en relación con la alfabetización informacional.

La fase final fue denominada como "Nuevo esclarecimiento y diagnóstico de la situación problemática”: diseño del plan de acción. En esta línea investigativa, la implementación de los tres talleres dio pie para aplicar uno más, donde se pudieran abordar los siete elementos ALFIN, en un escenario en que la población contara con bases y elementos que les permitiera consolidar e interiorizar las dinámicas de la alfabetización informacional. Para el caso en mención, la temática planteada del taller n. ${ }^{\circ} 4$ fue redes sociales y, por la situación cíclica del trabajo investigativo, quedó para ser aplicada en el futuro por el docente del área Tic y ambientes de aprendizaje en una de las licenciaturas que la UPTC oferta semestralmente.

Después de todos los análisis, donde en efecto había una falencia en la forma de abordar información con el elemento ético de por medio, se percibió la posibilidad de abordar con planes de estudio producto del análisis riguroso con estudiantes y personal administrativo el 
esclarecimiento del problema y, en consecuencia, se presenta el plan de acción ajustado a esos hallazgos. El plan se diseñó bajo los referentes ${ }^{32}$ donde se plantean los siguientes elementos: objetivo, metas, indicadores, acciones, recursos, responsable y tiempo.

\section{Conclusiones}

La ética es la categoría emergente de este trabajo; su empleo acertado en la información empodera a las sociedades para que construyan conocimiento. Así mismo, la importancia del acceso a la información, la evaluación y el uso ético, se logran vinculando la aplicación de ciertas habilidades que facultan a los usuarios en la forma de acceder y utilizar tan diversa y prolija información de la manera más eficiente.

Se tuvo en cuenta lo expuesto por Roxana Morduchowicz ${ }^{33}$, en cuanto a que, si bien los estudiantes que hacen parte de esta revolución digital se desenvuelven con habilidad en estos entornos, algunos son "ingenuos digitales" y son presa fácil de quienes comparten información sesgada.

En gran parte del estado del arte de este estudio se observa el plan ético como turbina transformadora en el acceso a la información fiable. Es este importante elemento el que habilita las sociedades en defensa de la información que genera dinámicas de empoderamiento y proyecciones de enriquecimiento intelectual a la humanidad.

El andamiaje de ese proyecto se articuló, en cuanto al plan de acción, en función de acceder a contenido digital fiable; se infiere que, mediados por este, se vislumbra una oportunidad de transformación en el análisis de información veraz como una práctica que se debe privilegiar en el aula de clase.

En lo que respecta al objetivo del diagnóstico, se concluye que hay debilidades que no permiten que en algunos estudiantes el aspecto ético se maneje con un grado de efectividad satisfactorio y uno de los acuerdos en común es la falta de conocimiento informacional.

Con relación al segundo objetivo, los talleres se realizaron buscando reforzar competencias de elementos de la ALFIN. Esto condujo a la detección de una falencia en la manera de abordar información desde la mirada ética, generando a su vez la presentación del plan de acción ajustado a esos hallazgos.

Por último, en lo concerniente a los contenidos digitales confiables entre la población objeto de estudio, cabe mencionar que todo es un proceso de ires y venires, en el que, en principio, se pueden entender los errores cometidos por la falta de experticia.

\section{Conflicto de interés}

Los autores declaran que no tienen conflicto de interés.

32 Víctor Gutiérrez, "Plan de acción", 2008.

33 Morduchowicz, Ruidos en la web. 


\section{Financiamiento}

El proyecto es un trabajo de maestría que se trabajo desde el grupo de investigación CETIN, pero cabe aclarar que no tuvo ninguna financiación.

\section{Referencias}

Aguaded, Ignacio, Isidro Marín y Mari Carmen Caldeiro, "Desarrollo de la competencia mediática en el contexto iberoamericano", Revista Letral, n. ${ }^{\circ} 20,2018$ : 156-182. http://revistaseug.ugr index.php/ letral/article/view/7814/6847

Andrada, Pablo, "Estado de la cuestión de la formación docente en educación en medios", Comunicación y Medios n. ${ }^{\circ}$ 31, 2015: 83-96. https://auroradechile.uchile.cl/index.php/RCM/article/ view/36700/39652

Castañeda-Peña, Harold, Liliana González, Gloria Marciales, Jorge Barbosa y Juan Barbosa, “Recolectores, verificadores y reflexivos: perfiles de la competencia informacional en estudiantes universitarios de primer semestre", Revista Interamericana de Bibliotecnología, vol. 33, n. ${ }^{\circ} 1,2010$. http://www. scielo.org.co/scielo.php?pid=S0120-09762010000100008\&script=sci_arttext\&tlng=pt

Chisaba, María, César Hernández y Marisol Ramírez. "El taller como estrategia metodológica para el aprendizaje de expresiones idiomáticas del inglés en niños de cuarto de primaria. Tesis de grado de licenciatura en Educación Básica con énfasis en Humanidades, Universidad Libre, 2013. https://repository.unilibre.edu.co/bitstream/handle/10901/8210/TRABAJO\%20DE\%20GRADO. pdf?sequence=1\&isAllowed=y

Denzin, Norman, ed. Sociological Methods. A Sourcebook. Chicago: Aldine Publishers, 1970.

Ferrés, Joan, “La competencia en comunicación audiovisual: dimensiones e indicadores", Comunicar, vol. 29, n. 15,2007 : 100-107.

Flores, Carolina; Roig, Rosabel. “Factores personales que inciden en la autovaloración de futuros maestros sobre la dimensión pedagógica del uso de TIC" Revista iberoamericana de educación superior, vol. 10, n. .27, 2019. https://www.redalyc.org/articulo.oa?id=299159574008

Gil, Javier, "La estadística en la investigación educativa", Revista de Investigación Educativa, vol. 21, n. , 2003: 233. https://revistas.um.es/rie/article/view/99191/94791

Gómez, Francisco, “Desinformación en Internet y hegemonía en redes sociales”, Gestión de las Personas y Tecnología, vol. 5, n. ${ }^{\circ}$ 16, 2013: 26-34. https://dialnet.unirioja.es/servlet/articulo?codigo=4759721

Gutiérrez, Victor, “Plan de acción”, 2008. https://es.scribd.com/doc/6490905/Plan-de-Accion

Lara, Pablo, “Uso de contenidos digitales: tecnologías de la información, sociedad del conocimiento y universidad", Revista de Universidad y Sociedad de Conocimiento, vol. 2, n.o 2, 2005: 1-5. http:// www.redalyc.org/pdf/780/78020107.pdf

Lau, Jesús, “Conceptual Relationship of Information Literacy and Media Literacy”. En Conceptual Relationship of Information Literacy and Media Literacy in Knowledge Societies. 76-91. París: UNESCO, 2013.

Lee, Alice y Clement So, "Alfabetización mediática y alfabetización informacional: similitudes y diferencias". Comunicar, vol. 21, n. ${ }^{\circ}$ 42, 2014: 137-146. http://dx.doi.org/10.3916/C42-2014-13

Lewin, Kurt, “Acción-investigación y problemas de las minorías”, Revista de Psicología Social, vol. 3, n. ${ }^{\circ}, 1988$.

Ministerio de Educación Nacional, Competencias TIC para el desarrollo profesional docente, Bogotá: Imprenta Nacional, 2013. https://www.mineducacion.gov.co/1759/articles- 39097_archivo_pdf_ competencias_tic.pdf 
Morduchowicz, Roxana. Ruidos en la web. Cómo se informan los adolescentes en la era digital. Buenos Aires: Ediciones B, 2018.

Naval, Concepción y Elena Arbués, "La alfabetización mediática de los futuros profesores de educación primaria y secundaria". En Sociedad del conocimiento y educación, coord. Lorenzo García. Madrid: 2012. https://www.researchgate.net/profile/Lorenzo_Garcia-Aretio2/publication/234646907_Sociedad_del_conocimiento_y_educacion/links/0912f50fed876df407000000.pdf\#page=93

Partnership for 21st century skills (P21), “Framework definitions”, 2009. http://www.p21.org/storage/ docu- ments/P21_Framework_Definitions.pdf

Pérez, M. ${ }^{a}$ Amor y Águeda Delgado, “De la competencia digital y audiovisual a la competencia mediática: dimensiones e indicadores", Comunicar, vol. 20, n. ${ }^{\circ}$ 39, 2012: 25-34. doi:10.3916/C39,2012-02-02

Ruiz, Enrique, “Desarrollo de contenidos digitales educativos en comunidades de aprendizaje", http:// bibliotecadigital.conevyt.org.mx/colecciones/documentos/somece2002/Grupo2/Ruiz.pdf

Sáenz, Francisco J., “Alfabetización Informacional: qué es y cómo se adquiere InVito, el blog de la Biblioteca Francisco de Vitoria., https://diarium.usal.es/vito/2013/10/02/ alfabetizacion-informacional-que-es-y-como-se-adquiere/

Tirado, Alejandro y María Pinto, "75 lecciones aprendidas en programas de alfabetización informacional en universidades iberoamericanas", Revista española de Documentación Científica, vol. 37, n. ${ }^{\circ}$, 2014. http://dx.doi.org/10.3989/redc.2014.3.1118

Wilson, Carolyn, Alton Grizzle, Ramon Tuazon, Kewame Akyempong y Chi Kim Cheung, Alfabetización Mediática e Informacional: Currículum para profesores. París: UNESCO, 2011. https://unesdoc. unesco.org/ark:/48223/pf0000216099 